\section{mTOR caveats}

\section{By Michael J. Haas, Senior Writer}

In many tumor types, rapamycin is thought to inhibit mammalian target of rapamycin's regulation of a key transcription factor-hypoxiainducible factor- $1 \alpha$-that is involved in tumor cell growth and proliferation. A widely used biomarker therefore relies on suppression of this factor as a proxy for mammalian target of rapamycin inhibition. However, a recent report in Current Biology questions whether that particular biomarker is appropriate in all tumor types.

Mammalian target of rapamycin (mTOR) is a serine/threonine protein kinase that regulates cell growth, proliferation and survival as well as protein synthesis and transcription. Hypoxia-inducible factor- $1 \alpha$ (HIF- $1 \alpha)$, an oxygen-sensitive transcription factor, sits downstream of and is regulated by mTOR. HIF- $1 \alpha$ is highly expressed in many tumor types and is known to regulate the expression of genes involved in tumor angiogenesis, metabolism and invasion.

Suppression of HIF- $1 \alpha$ in turn suppresses GLUT1, a glucose transport gene that regulates uptake of fluorodeoxyglucose (FDG). As a result, mTOR-induced suppression of HIF- $1 \alpha$ can be quantitated by monitoring the uptake of radiolabeled FDG with PET.

In the Jan. 8 issue of Current Biology, a research team led by Karen Cichowski found that in neurofibromatosis type 1 (NF1)-related tumors, rapamycin suppressed tumor growth without suppressing HIF- $1 \alpha$ expression-raising questions about the general relevance of HIF- $1 \alpha$ as a biomarker of mTOR inhibition. ${ }^{1}$ Cichowski is an assistant professor of medicine at Brigham and Women's Hospital's Department of Medicine and at Harvard Medical School. Other researchers on the paper were from the Dana-Farber Cancer Institute, Massachusetts General Hospital and Children's Hospital Boston.

Rapamycin, also called sirolimus, is a potent immunosuppressor that inhibits interleukin-2 (IL-2) activation of B cells and T cells. Rapamune sirolimus is marketed by Wyeth as an immunosuppressant to prevent organ transplant rejection.

NF1 is a genetic disease characterized by the development of malignant peripheral nerve sheath tumors (MPNSTs). Cichowski and others had already demonstrated that NF1 inactivation led to aberrant activation of mTOR pathways, suggesting that MPNSTs depend on increased mTOR signaling for growth., ${ }^{2,3}$ Thus, Cichowski's team sought to identify the effectors that mTOR activated for MPNST development.

The research team used a genetically engineered, spontaneous tumor model of NF1 in which mice deficient in both NF1 and p53 were treated with rapamycin. The scientists found that rapamycin did indeed suppress MPNST growth, but that it had no effect on the expression of HIF$1 \alpha$ or its transcripts. Instead, results showed that rapamycin suppressed tumor growth via suppression of mTOR complex-1 (TORC1), a cluster of three proteins-including mTOR-that functions as a nutrient and energy sensor to control protein synthesis.

The researchers looked for other activators of mTOR in MPNSTs among known mTOR targets: cyclin D1 (CCND1), programmed cell death-4 (PDCD4), c-myc, p27 and p21. Of these, rapamycin suppressed only CCND1 expression.

CCND1 is a member of the cyclin family, which regulate cyclindependent kinases. Mutations in the CCND1 gene have been observed in many tumors and are thought to contribute to tumorigenesis.

Additional in vitro experiments by Cichowski's team demonstrated that NF1 inactivation enhanced CCND1 expression, a finding that correlated with findings reported by other researchers. ${ }^{4}$

Taken together, these results indicated that CCND1 was a critical target of rapamycin in MPNSTs in vivo. This led Cichowski's team to conclude that CCND1 expression might be a better biomarker than HIF-1 $\alpha$ suppression for mTOR inhibition in those tumors.

But the team, noting that CCND1 had been implicated as a critical rapamycin target in mantle cell lymphoma, also suggested CCND1 as a general target of $\mathrm{MTOR}-$ and a biomarker of mTOR inhibition-in tumorigenesis.

Henrik Oerum, CSO of Santaris Pharma A/S, said the Current Biology study "underpins what is probably not a big surprise to most researchers: one cannot automatically assume that a particular drug elicits its effect in different cell types by exactly the same mechanism of action. In the case of MPNSTs, the study suggests that cyclin D1 could be an appropriate biomarker. However, much work remains to consolidate this observation, including target-disease association studies in human MPNST tumors."

Santaris and partner Enzon Pharmaceuticals Inc. are developing EZN-2968, a HIF-1 $\alpha$ antagonist in Phase I testing to treat lymphoma and solid tumors. "Clinical indications chosen for this drug candidate are all selected on the basis of scientific and medical data that links elevated levels of HIF- $1 \alpha$ to the pathology of the disease," Oerum said.

John Lyons, VP of translational biology at Astex Therapeutics Ltd., told $S c i B X$ that the paper supports "the need for a thorough, intimate understanding of the signaling networks in the target cell types. Without this information, costly clinical trials might prove ineffective and, more importantly, patients with MPNSTs and other cancers will not receive the targeted therapy that they deserve."

Last year, Astex completed a Phase I trial of its AT7519 CDK inhibitor in solid tumors. The company is considering whether to develop the compound for chronic lymphocytic leukemia. 
Behind AT7519, Astex has its AT9311 CDK inhibitor in preclinical development for multiple myeloma. AT9311 is partnered with Novartis AG.

Lyons suggested the findings "will certainly lead clinicians to put forward a case for testing new signal transduction inhibitors in patients with NF1-associated malignancies," including rapamycin analogs and CDK1, CDK2 and CDK4 inhibitors.

Although the question of which mTOR biomarker is suitable for which cancer will take time to sort out, another finding in Cichowski's paper could have more near-term implications. The researchers suggested that genetically engineered mouse models make better preclinical predictors of therapeutic responses to mTOR inhibitors in human tumors than xenograft tumor models.

In xenografts, mTOR inhibitors have been found to suppress tumors by rapidly disrupting tumor microvasculature-often leading to tumor regression. But in genetically engineered models, Cichowski and her team obtained very different results: after eight days of treatment with rapamycin, tumor growth was suppressed but the tumors still had clearly defined microvasculature. Rapamycin had no observable effect on the density of microvasculature until day 14 and beyond.

The researchers attributed the discrepancy to differences in signaling that give spontaneously arising tumors more stable microvasculature.

Oerum agreed that this was probably the case. "The effect of rapamycin on tumor vasculature is likely to be better portrayed in the engineered mouse," he said.

Oerum said the formation of a xenograft tumor involves the rapid development of crude vasculature that ties the tumor into the host's circulatory system and allows the xenograft to survive. Such "emergency vascularization," he said, might be highly susceptible to disruption by a drug.

A spontaneously arising tumor-such as that in a genetically engineered mouse or a human patient with NF1-develops vasculature over time, as the tumor grows in the host. Such a tumor is likely to have more stable and less readily disrupted vasculature than a xenograft.

"We used various xenografts for our HIF- $1 \alpha$ work," Oerum told $S c i B X$. "We would have loved, though, to use genetically engineered models if they had been available to us."

Lyons agreed. "Xenograft models are the workhorses of the cancer therapy development field," he said. "But the transgenic models of knockouts and knockins are much more relevant to a patient's disease."

Cichowski was unable to respond to requests for an interview.

\section{REFERENCES}

1. Johannessen, C. et al. Curr. Biol.; published online Dec. 27, 2007; doi:10.1016/j.cub.2007.11.066

Contact: Karen Cichowski, Genetics Division, Department of Medicine, Brigham and Women's Hospital, Boston, Mass. e-mail: kcichowski@rics.bwh.harvard.edu

2. Dasgupta, B. et al. Cancer Res. 65, 2755-2760 (2005)

3. Johannessen, C. et al. Proc. Natl. Acad. Sci. USA 102, 8573-8578 (2005)

4. Kim, H. et al. J. Neurosci. 21, 1110-1116 (2001)

COMPANIES AND INSTITUTIONS MENTIONED

Astex Therapeutics Ltd., Cambridge, U.K.

Brigham and Women's Hospital, Boston, Mass.

Children's Hospital Boston, Boston, Mass.

Dana-Farber Cancer Institute, Boston, Mass.

Enzon Pharmaceuticals Inc. (NASDAQ:ENZN), Bridgewater, N.J.

Harvard Medical School, Boston, Mass.

Massachusetts General Hospital, Boston, Mass.

Novartis AG (NYSE:NVS; SWX:NOVN), Basel, Switzerland

Santaris Pharma A/S, Horsholm, Denmark

Wyeth (NYSE:WYE), Madison, N.J. 